\title{
The interfering effects of frequent auditory verbal hallucinations on shadowing performance in schizophrenia.
}

Sir

Currently, two broad theoretical approaches to auditory verbal hallucinations (AVH) in schizophrenia are recognized (Jones, 2010). The 'neurological' model proposes that they are essentially perceptual in nature, arising from pathological overactivity in brain systems devoted to auditory perception. In contrast, the 'cognitive’ model maintains that they are due to a failure to recognize internal, non-perceptual stimuli for example, inner speech, mental imagery or intrusive memories - as being selfgenerated. While the latter approach has been tested in a variety of experimental paradigms (Waters et al., 2012), the main support for the neurological model is circumstantial, coming from clinical observations that auditory phenomena, up to and including people speaking, are sometimes can be seen in patients with epilepsy (Bisulli et al., 2004) or elicited by electrical stimulation of the superior temporal gyrus in patients undergoing neurosurgery (Penfield and Perot, 1963).

At the same time, the neurological model does make a testable prediction: this is that if AVH are perceptual in nature then their presence at high frequency should interfere with the performance of tasks that make competing auditory-perceptual demands. To test this, we sought patients from five psychiatric hospitals in Barcelona and Zaragoza who reported experiencing near-continuous AVH ( $\mathrm{N}=22$; age 41.31 \pm 8.60 ; m/f 17/5; premorbid IQ estimated using a word pronunciation test (Gomar et al., 2011) 100.38 \pm 8.27 ). Subjective voice frequency was confirmed by asking the patients to tap each time they experienced a voice during a 5-minute period; any who tapped less than 5 times were excluded (12 tapped between 5 and 20 times; 5 between 21 and 49 times; and 550 or more times). A comparison group of patients who currently 
experienced AVH less than once a week $(\mathrm{N}=28$; age 43.29 \pm 11.47 ; m/f 17/11; estimated premorbid IQ 100.29 \pm 8.96 ) were also tested, as were healthy volunteers

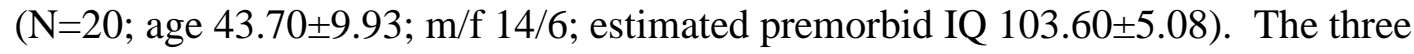
groups were matched for age $(\mathrm{F}=0.34 ; \mathrm{p}=0.71)$, sex $\left(\mathrm{Chi}^{2}=1.59 ; \mathrm{p}=0.45\right)$ and estimated premorbid IQ $(\mathrm{F}=1.25 ; \mathrm{p}=0.29)$. The patients all met DSM-5 criteria for schizophrenic/schizoaffective disorder and were all on drug treatment. All participants gave written informed consent and the study was approved by the local research ethics committee.

The participants listened to 12 passages of speech that were presented binaurally through headphones and were instructed to repeat them verbatim as they heard them. Half of the passages were short narratives (taken from two memory batteries: the Rivermead Behavioural Memory Test (RBMT) (Wilson et al., 1985) and the Wechsler Memory Scale (WMS-III) (Wechsler, 2000). All stories were read by the same speaker; they had a length of 62-90 words (mean = 70.50) and lasted 24-36 seconds $($ mean $=28.33)$. The other 6 passages consisted of short conversations between two speakers that dealt with the same topics as the stories. The dialogues had a length of 81-120 words (mean $=92.67)$ and a duration of 25-38 seconds (mean $=$ 29.83). Responses were recorded onto a laptop. Scoring was made by three independent raters, who were blind to the participant's group and diagnosis and for whom good inter-rater reliability had previously been established.

Overall shadowing performance is shown in Figure 1. There were significant differences between groups (Kruskal-Wallis $\mathrm{Chi}^{2}=16.09, p<0.001$ ): both groups of patients performed significantly more poorly than the healthy controls (Mann- 
Whitney, $\mathrm{p}=0.001$ in both cases). However, performance did not differ significantly between the patients with and without AVHs (Mann-Whitney, $\mathrm{p}=0.95$ ).

[Figure 1about here] Figure 1. Shadowing task performance for the three groups. Vertical axis indicates percent correctly repeated words (HC, healthy controls, AVH+, patients with frequent hallucinations, AVH-, patients with no current hallucinations).

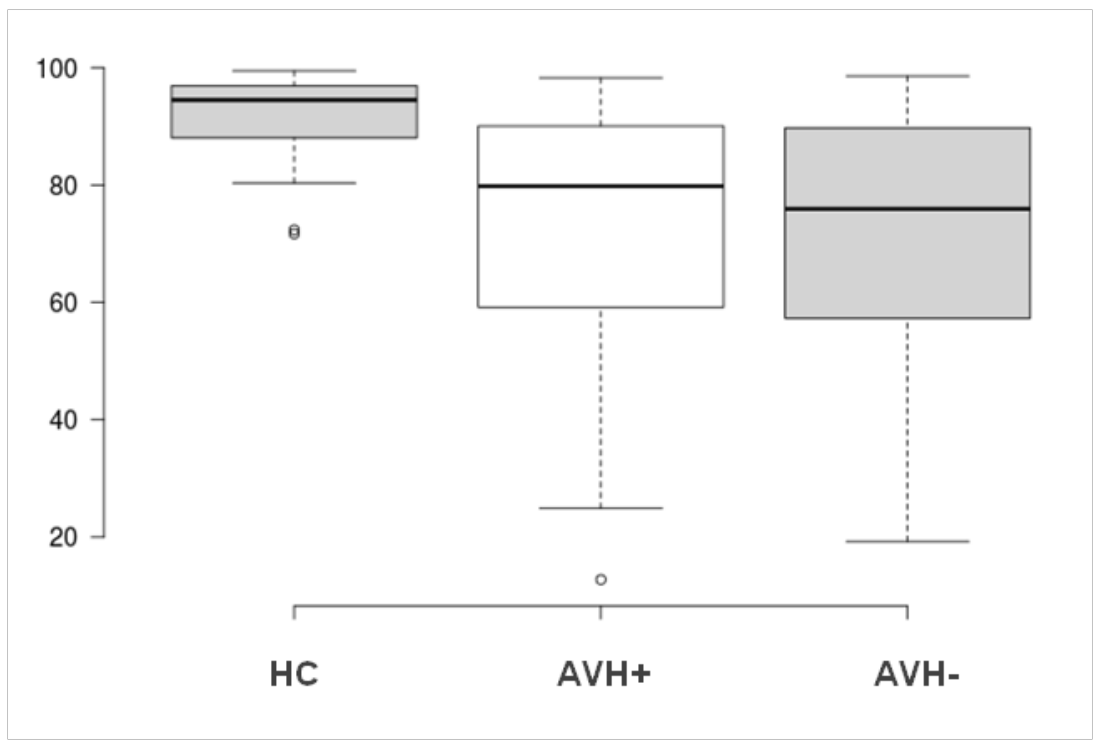

Significant differences between the patient groups did not emerge when the narrative and dialogues conditions were considered separately. Voice frequency in the hallucinating patients, as measured by the tapping test, was not significantly associated with shadowing performance ( $\rho=.17, p=0.46)$.

Our results represent, as far as we know, the first attempt to directly test the neurological model of AVH in schizophrenia, and they strikingly fail to provide support for it. It does not seem likely that this negative finding was due to the task used - the disruptive effects of irrelevant auditory material on shadowing performance is well-recognized, for example forming the basis of the widely used the dichotic listening task. Nor does it seem credible that the frequency of voices the 
patients experienced was insufficient to interfere with shadowing performance $-45 \%$ of the sample of hallucinators experienced voices at a frequency of $\geq 20$ times/minute. Also arguing against this interpretation is the fact that there was no significant correlation between shadowing performance and hallucination frequency as measured by tapping. It remains possible that the act of speaking during the shadowing task somehow acted to suppress the patients' voices; however, the evidence that such a manoeuvre actually reduces auditory hallucinations is not strong (Bick and Kinsbourne, 1987; Gallagher et al., 1994; Green and Kinsbourne, 1990).

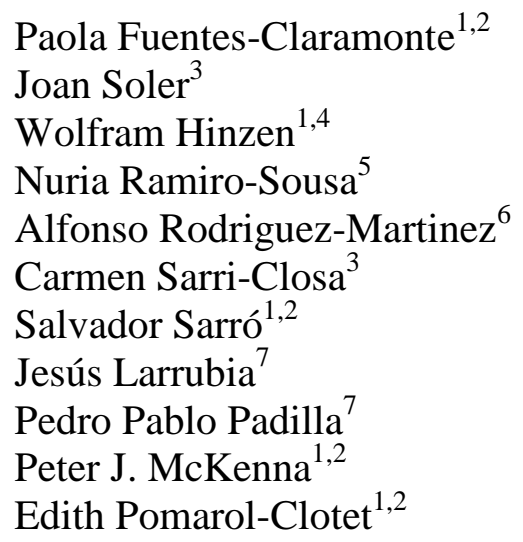

1. Fundació per a la Investigació i la Docència Maria Angustias Giménez (Barcelona, Spain)

2. CIBERSAM (Centro de Investigación Biomédica en Red de Salud Mental, Spain)

3. Benito Menni Complex Assistencial de Salut Mental (Barcelona, Spain)

4. Catalan Institute for Advanced Studies and Research (ICREA), and Department of Translation and Language Sciences, Universitat Pompeu Fabra, Barcelona, Spain.

5. Hospital de Sant Rafael (Barcelona, Spain)

6. Parc de Salut Mar (Barcelona, Spain)

7. Centro Neuropsiquiátrico N.S. del Carmen (Zaragoza, Spain)

Address for correspondence: Dr P J McKenna, FIDMAG Germanes Hospitalàries Research Foundation, C/ Dr. Antoni Pujadas, 38, E-08830 Sant Boi de Llobregat, Barcelona, Spain. email: mckennapeter1@gmail.com

All authors declare that they have no conflicts of interest. Financial support: Catalonian Government (2017-SGR-1271 and 2017-SGR-1265 to FIDMAG); Miguel Servet Research Contract (CPII16/00018 to EP-C), Juan de la Cierva-formación Contract (FJCC-2015-02285) and Research Project Grants (PI14/01148 to EP-C, PI14/01691 to P.M. and FFI2016-77647-C2 to WH). WH was additionally supported by the Ministerio de Economía y Competitividad (MINECO, Spanish Government, grants FFI2013-40526P, FFI 042177665-77665-4-16). 


\section{References}

Bick, P.A., Kinsbourne, M., 1987. Auditory hallucinations and subvocal speech in schizophrenic patients. Am. J. Psychiatry 144, 222-225.

Bisulli, F., Tinuper, P., Avoni, P., Striano, P., Striano, S., d'Orsi, G., Vignatelli, L., Bagattin, A., Scudellaro, E., Florindo, I., Nobile, C., Tassinari, C.A., Baruzzi, A., Michelucci, R., 2004. Idiopathic partial epilepsy with auditory features (IPEAF): a clinical and genetic study of 53 sporadic cases. Brain 127, 1343-1352.

Gallagher, A.G., Dinan, T.G., Baker, L.J., 1994. The effects of varying auditory input on schizophrenic hallucinations: a replication. Br. J. Med. Psychology 67, 67-75. Gomar, J.J., Ortiz-Gil, J., McKenna, P.J., Salvador, R., Sans-Sansa, B., Sarro, S., Guerrero, A., Pomarol-Clotet, E., 2011. Validation of the Word Accentuation Test (TAP) as a means of estimating premorbid IQ in Spanish speakers. Schiz. Res. 128, 175-176.

Green, M.F., Kinsbourne, M., 1990. Subvocal activity and auditory hallucinations: clues for behavioral treatments? Schiz. Bull. 16, 617-625.

Jones, S.R., 2010. Do we need multiple models of auditory verbal hallucinations? Examining the phenomenological fit of cognitive and neurological models. Schiz. Bull. 36, 566-575.

Penfield, W., Perot, P., 1963. The Brain's Record of Auditory and Visual Experience. A Final Summary and Discussion. Brain 86, 595-696.

Waters, F., Allen, P., Aleman, A., Fernyhough, C., Woodward, T.S., Badcock, J.C., Barkus, E., Johns, L., Varese, F., Menon, M., Vercammen, A., Laroi, F., 2012. Auditory hallucinations in schizophrenia and nonschizophrenia populations: a review and integrated model of cognitive mechanisms. Schiz. Bull. 38, 683-693.

Wechsler, D., 2000. Escala de memoria de Wechsler. WMS-III. TEA, Madrid. Wilson, B.A., Cockburn, J., Baddeley, A.D., 1985. The Rivermead Behavioural Memory Test (RBMT). Thames Valley Test Co, Reading, UK. 